\title{
Palmoplantar pustulotic arthro-osteitis of the peripheral joints with no sternocostoclavicular lesions
}

\author{
Michito Takagi, Jyunji Oda, Nobuyuki Tsuzuki, Hidekichi Sonozaki
}

Department of

Orthopaedic Surgery,

Saitama Medical Centre,

1981 Tsujido-Cho,

Kamoda, Kawagoe City,

Saitama Prefecture 350,

Japan

M Takagi

J Oda

N Tsuzuki

Department of

Orthopaedic Surgery,

Tokyo Metropolitan

Otsuka Hospital,

2-8-1 Minamiotsuka,

Toshima-Ku, Tokyo 170,

Japan

H Sonozaki

Correspondence to:

Dr Michito Takag

Department of

Saitama Medical Centre,

1981 Tsujido-Cho,

Kamoda, Kawagoe City,

Saitama Prefecture 350,

Japan.

Accepted for publication 16 July 1991
Orthopaedic Surgery,

\begin{abstract}
A 45 year old woman presented with swelling and pain on joint movement of her knees, although joint movement was relatively well maintained. Five years previously the patient had been diagnosed as having pustulosis palmoplantaris by a dermatologist. Treatment with non-steroidal anti-inflammatory drugs (NSAIDs) had no effect, but treatment with $5 \mathrm{mg} /$ day prednisolone caused remission of both the joint pain and swelling and the pustulosis palmoplantaris. There were no sternocostoclavicular lesions at any time during treatment. Clinical findings in this patient were different from previous reports and she was diagnosed as having palmoplantar pustulotic arthro-osteitis affecting the peripheral joints.
\end{abstract}

Pustulosis palmoplantaris is a skin disease of unknown aetiology characterised by sterile pustules on the palms and soles of the feet. Pustulotic arthro-osteitis occurs in about $10-30 \%$ of patients with palmoplantaris pustulosis. Its principal lesion consists of intersternocostoclavicular ossification, ${ }^{12}$ and lesions also often occur in the spinal column, sacroiliac, and peripheral joints. ${ }^{3}$ There have, however, been few reports of patients in whom the main lesion is peripheral arthritis without any sternocostoclavicular lesion. We report a patient with pustulosis palmoplantaris associated with peripheral arthritis in which the diagnosis

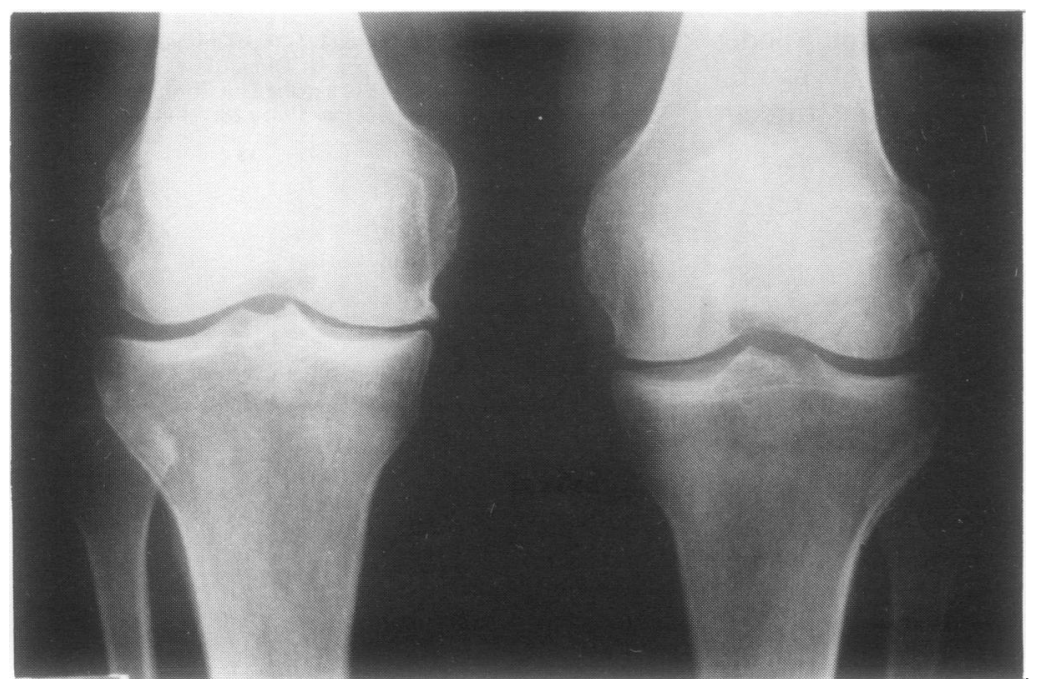

Figure 1 Plain radiograph of knee joints showing narrowing of the joint spaces and formation of bony spurs. was considered to be palmoplantar pustulotic arthro-osteitis of the peripheral joints.

\section{Case report}

The patient was a 45 year old woman who came to our clinic in June 1988 with swelling and pain on joint movement. The patient had had swelling of the right knee and pain while kneeling or sitting on her legs for two years before her initial visit to our clinic. Similar symptoms developed in the left knee four months before her initial visit.

The patient had no history of psoriasis, nor was there any history or clinical evidence of psoriasis in her family. She was $158 \mathrm{~cm}$ tall and weighed $57 \mathrm{~kg}$.

General physical examination showed slight pallor of the palpebral conjunctivae. There were no abnormal findings in the chest or abdomen, and no lymph node enlargement was detected. A chest radiograph showed no hilar lymphadenopathy.

Physical examination of the affected areas showed diffuse swelling of the knees bilaterally and pain on joint movement; however, the range of joint motion was relatively well maintained.

Five years before developing gonitis, pustules appeared on the patient's palms and soles, and she was diagnosed by a dermatologist as having pustulosis palmoplantaris. She was treated with steroid ointment.

In May 1989 the patient first noticed swelling and pain when she moved her wrists and ankles, and in July 1989 the same symptoms also developed in her elbows.

In spite of continuous treatment of the arthritis at these sites with non-steroidal antiinflammatory drugs (NSAIDs), the swelling and pain persisted. During that time, we did not observe any swelling or pain in the sternocostoclavicular region, nor any spondylitis nor sacroiliitis.

In November 1989 treatment with $5 \mathrm{mg} /$ day prednisolone by mouth was begun. After about two weeks of treatment, the swelling and pain in the joints throughout the body and the pustulosis palmoplantaris remitted; they are still in remission at the time of writing.

A plain radiograph showed the formation of bony spurs and mild bilateral narrowing of the knee joint spaces (fig 1); however, no ossification of the sternocostoclavicular region (fig 2), and no bone erosions of the knees, hands, ankles, or elbows were seen.

A technetium-99m diphosphonate labelled scintigram of the bones showed an increased 


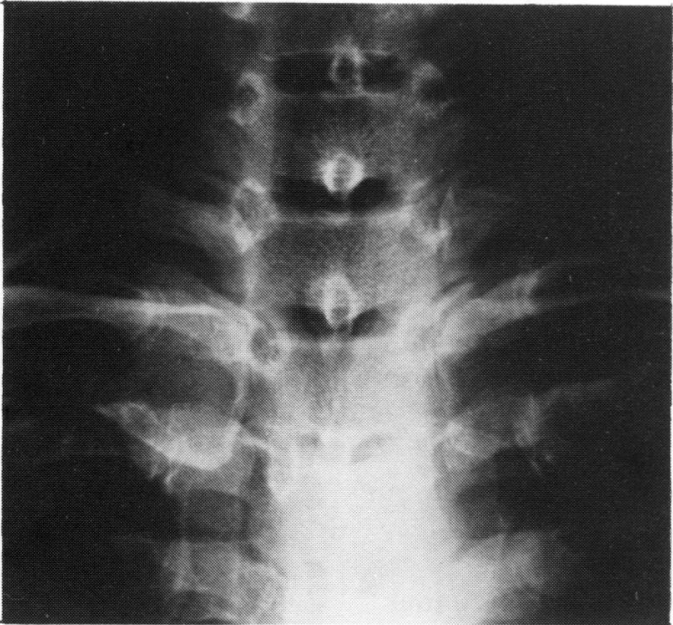

Figure 2 Plain radiograph of the sternocostoclavicular region showing no marked ossifications in this region.

uptake of the isotope in the knees and ankles bilaterally, but no increased uptake was seen in the sternocostoclavicular region (fig 3).

Bacteriological examination of the knee joint fluid gave negative results.

Haematological tests conducted in June 1988 gave the following results: erythrocyte sedimentation rate $79 \mathrm{~mm} / \mathrm{h}$, erythrocyte count $3570 \times 10^{9} / 1$ haemoglobin $99 \mathrm{~g} / 1$, leucocyte count $8.2 \times 10^{9} / 1$ (neutrophils $51 \%$, eosinophils $0 \%$, basophils $2 \%$, lymphocytes $47 \%$ ), platelets $454 \times 10^{9} / 1$, total protein $74 \mathrm{~g} / 1$ (normal $65-80$ ), albumin $36 \mathrm{~g} / \mathrm{l}$ (normal 37-50), albumin/ globulin 0.95 , latex fixation test negative, Waaler-Rose reaction $<40$ (normal $<40$ ), anti-

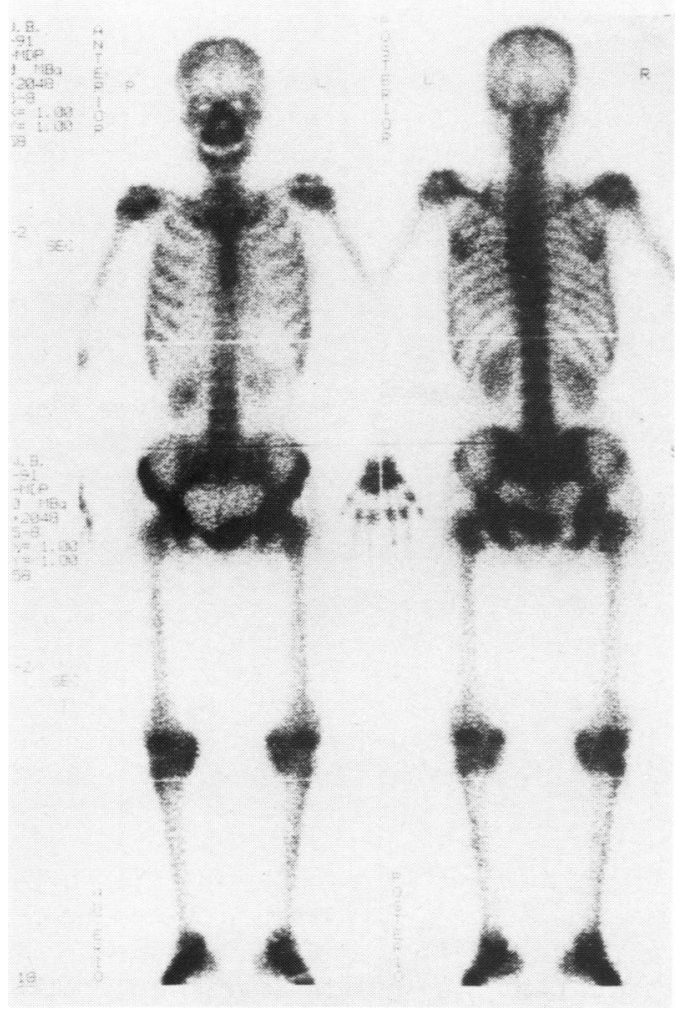

Figure 3 Technetium-99m diphosphonate labelled bone scan showing increased uptake in the knees and ankles, but no increased uptake in the sternocostoclavicular region. bodies to streptolysin $\mathrm{O} 81 \mathrm{IU} / \mathrm{ml}$ (normal $<140$ ), C reactive protein $88 \mathrm{mg} / \mathrm{l}$ (normal $<10$ ), Wassermann reaction negative, antinuclear antibodies negative, IgG $19.4 \mathrm{~g} / \mathrm{l}$ (normal 10-17), IgA 4.87 g/l (normal $1 \cdot 7-2 \cdot 9$ ), IgM $2 \cdot 27 \mathrm{~g} / \mathrm{l}$ (normal $0 \cdot 9-2 \cdot 1$ ), HLA typing A2, Bw61, Bw62, and Cw3. Electrolytes, liver, kidney, and thyroid function tests were all normal, as was the urine analysis.

\section{Discussion}

The presence of the principal lesion in the sternocostoclavicular region has traditionally been regarded as characteristic of arthro-osteitis accompanying pustulosis palmoplantaris. Although there have been cases of peripheral arthro-osteitis, most have been accompanied by lesions in the sternocostoclavicular region. Peripheral arthro-osteitis is characteristically regarded as being transient and not inducing contracture deformities. In addition, NSAIDs have been considered more effective than adrenocortical steroids. ${ }^{4}$

This paper reports a patient with pustulosis palmoplantaris who had multiple peripheral arthritis unaccompanied by lesions in the sternocostoclavicular region, spinal column, or sacroiliac joints. The arthritis was not transient, but persisted for four years with little remission, failing to respond to NSAIDs.

Patterson and Bentley-Corbett reported a case of palmoplantar pustulotic arthro-osteitis in which peripheral arthritis was predominant. ${ }^{5}$ Their patient was reported to have persistent gonitis for which adrenocortical steroids and radiation treatment were more effective than NSAIDs. Although our patient is similar to theirs in these respects, there are some differences in that the peripheral arthritis in their patient affected just a single joint and was accompanied by lesions in the sternocostoclavicular region and spinal column.

Hoshino and Minakami reported a patient with pustulosis palmoplantaris complicated by rheumatoid arthritis. ${ }^{6}$ The patient suffered polyarthritis persisting for four years in association with pustulosis palmoplantaris, but no lesions of the sternocostoclavicular region or spinal column were present. Plain radiographs showed erosion of the carpal and interphalangeal joints. Rheumatoid factor was positive. Our patient is similar in that polyarthritis was present, but lesions of the sternocostoclavicular region, spinal column, and sacroiliac joints were absent. No morning stiffness was observed in our patient and the lesions of the metacarpophalangeal and metatarsophalangeal joints often seen in patients with rheumatoid arthritis were not detected. Moreover, a plain radiograph showed not bone erosions, but bony spurs. Rheumatoid factor was also negative. In these respects, our patient is different from the patient reported by Hoshino et al. ${ }^{6}$

Although our patient had arthro-osteitis accompanying pustulosis palmoplantaris, as the clinical findings are different from those of typical palmoplantar pustulotic arthro-osteitis, palmoplantar pustulotic arthro-osteitis with predominant peripheral arthritis, and pustulosis 
palmoplantaris complicated by rheumatoid arthritis, we diagnosed this patient as having palmoplantar pustulotic arthro-osteitis affecting the peripheral joints.

There have been few reports of such patients, but they may not be rare. As the number of reports of such patients increases, it is probable that palmoplantar pustulotic arthro-osteitis will be classified into various subtypes. We have reported this patient, in part, with the intention of encouraging further reports of such cases in the future.
1 Sonozaki H, Kawashima M, Hongo O, et al. Incidence of arthro-osteitis in patients with pustulosis palmaris et plantaris. Ann Rheum Dis 1981; 40: 554-7.

2 Jurik A G, Ternowitz T. Frequency of skeletal disease, arthro-osteitis, in patients with pustulosis palmoplantaris. I Am Acad Dermatol 1988; 18: 666-71.

3 Sonozaki $\mathrm{H}$, Mitsui $\mathrm{H}$, Miyanaga $\mathrm{Y}$, et al. Clinical features of 53 cases with pustulotic arthro-osteitis. Ann Rheum Dis $1981 ; 40$ : 547-53.

4 Sonozaki H, Azuma A, Okai K, et al. Arthro-osteitis associated with pustulosis palmaris et plantaris. The Ryumachi 1980; 20: 132-9 (In Japanese with English abstract).

5 Patterson A C, Bentley-Corbett K. Pustulotic arthroosteitis. 7 Rheumatol 1985; 12: 611-4.

6 Hoshino T, Minakami M. Rheumatoid arthritis associated

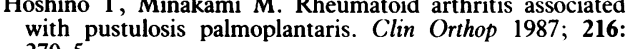
$270-5$. 